\title{
Evaluating a computer aid for assessing stomach symptoms (ECASS): study protocol for a randomised controlled trial
}

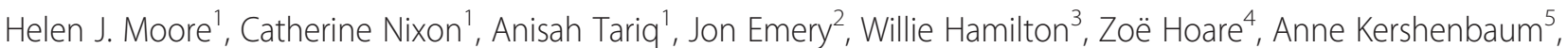
Richard D. Neal ${ }^{6}$, Obioha C. Ukoumunne ${ }^{7}$, Juliet Usher-Smith ${ }^{5}$, Fiona M. Walter ${ }^{5}$, Sophie Whyte ${ }^{8}$ and Greg Rubin ${ }^{{ }^{*}}$

\begin{abstract}
Background: For most cancers, only a minority of patients have symptoms meeting the National Institute for Health and Clinical Excellence guidance for urgent referral. For gastro-oesophageal cancers, the 'alarm' symptoms of dysphagia and weight loss are reported by only 32 and $8 \%$ of patients, respectively, and their presence correlates with advanced-stage disease. Electronic clinical decision-support tools that integrate with clinical computer systems have been developed for general practice, although uncertainty remains concerning their effectiveness. The objectives of this trial are to optimise the intervention and establish the acceptability of both the intervention and randomisation, confirm the suitability and selection of outcome measures, finalise the design for the phase III definitive trial, and obtain preliminary estimates of the intervention effect.
\end{abstract}

Methods/design: This is a two-arm, multi-centre, cluster-randomised, controlled phase II trial design, which will extend over a 16-month period, across 60 general practices within the North East and North Cumbria and the Eastern Local Clinical Research Network areas. Practices will be randomised to receive either the intervention (the electronic clinical decision-support tool) or to act as a control (usual care). From these practices, we will recruit 3000 adults who meet the trial eligibility criteria and present to their GP with symptoms suggestive of gastrooesophageal cancer. The main measures are the process data, which include the practitioner outcomes, service outcomes, diagnostic intervals, health economic outcomes, and patient outcomes. One-on-one interviews in a subsample of 30 patient-GP dyads will be undertaken to understand the impact of the use or non-use of the electronic clinical decision-support tool in the consultation. A further 10-15 GPs will be interviewed to identify and gain an understanding of the facilitators and constraints influencing implementation of the electronic clinical decisionsupport tool in practice.

Discussion: We aim to generate new knowledge on the process measures regarding the use of electronic clinical decision-support tools in primary care in general and to inform a subsequent definitive phase III trial. Preliminary data on the impact of the support tool on resource utilisation and health care costs will also be collected.

Trial registration: ISRCTN Registry, ISRCTN12595588.

Keywords: Primary care, General practice, Oesophageal cancer, Gastric cancer, Electronic clinical decision support

\footnotetext{
* Correspondence: g.p.rubin@durham.ac.uk

${ }^{1}$ School of Medicine, Pharmacy \& Health, Durham University, Wolfson

Research Institute, Queens Campus, Thornaby TS17 6BH, UK

Full list of author information is available at the end of the article
} 


\section{Background}

For most cancers, only a minority of patients have symptoms that meet the National Institute for Health and Clinical Excellence (NICE) guidance for urgent referral $[1,2]$. For gastro-oesophageal (G-O) cancers, the 'alarm' symptoms of dysphagia and weight loss are reported by only 32 and $8 \%$ of patients, respectively [3], and their presence correlates with advanced-stage disease [4]. Several other symptoms predict G-O cancer but with absolute risks in the region of $1 \%$. Not surprisingly, therefore, delays in diagnosis may occur; $25 \%$ of oesophageal and $36 \%$ of gastric cancer patients visit their GPs three times or more before diagnosis [5]; only 34 and $23 \%$, respectively, are referred by the 2-week wait (2WW) pathway for urgent referral of patients with suspected cancer; and 22 and $33 \%$, respectively, present as emergencies [6].

The primary investigation for $\mathrm{G}-\mathrm{O}$ cancer is gastroscopy, whether by $2 \mathrm{WW}$ or direct-access referral. One solution to a diagnostic delay may be to investigate more patients. General practices in the lowest tertile of the gastroscopy referral rate (6.2/1000 per annum (p.a.)) have worse outcomes (emergency admissions, 6-month mortality) than practices in the highest tertile $(16.4 / 1000$ p.a.) [7]. Patients 55 years of age and older account for $65 \%$ of all gastroscopies, and the gastroscopy rate in this age group is $17.5 / 1000$ p.a. [8]. Gastroscopy rates are much higher in mainland Europe [9], probably contributing to the observed better survival. However, because gastroscopy is a costly and invasive procedure, any increase in activity should target those patients most likely to benefit.

Recent studies, including those by the co-authors, have provided robust estimates of the risk of several cancers (including G-O) for the symptomatic patient in primary care [3, 10-15]. From these, risk-assessment tools (RATs) have been developed for use in general practice [16-18]. In a feasibility study, their use was associated with increases in $2 \mathrm{WW}$ referrals, use of investigations, and new cancer diagnoses [19]. They have now been developed in an electronic clinical decision support (eCDS) format by Macmillan Cancer Support using the BMJowned Informatica platform and integrated with some GP clinical computer systems.

The Macmillan eCDS tool covers six cancer sites (including G-O cancer). It utilises either of two diagnostic algorithms: the first is based on risk-assessment tools (RAT) [3], and the second, on Qcancer research [18]. Following extensive piloting, this tool was distributed as a National Awareness and Early Diagnosis Initiative (NAEDI) project to 500 practices in 15 Cancer Networks for a pilot period of 9 months), and its usability has been qualitatively evaluated in an internal report [20]. However, the evaluation of this project did not examine the impact of the eCDS in investigations, on GPs and patients in the consultation, on clinical outcomes, or on the health economics of its use.

Despite increasing promotion of the CDS for clinical practice, great uncertainty still exists about its effectiveness for potential cancer symptoms. One systematic review has identified the features critical to success of clinical decision-support interventions [21]. A second review, of computerised (eCDS) systems, found that they improved practitioner performance in $64 \%$ of the 97 included studies [22], whereas a third review identified prompt fatigue as a strong reason for failure of the eCDS [23]. No randomised controlled trial in primary care has reported on the eCDS for cancer diagnosis.

Important, but unanswered, questions relate to the implementation and cost-effectiveness of the cancer eCDS in primary care settings, the impact on clinical outcomes, and utility over time [24]. We propose to study this using the Macmillan eCDS tool (in its RAT version) for G-O cancer diagnosis [3] as our exemplar, for the clinical reasons listed above, for its health-economic and resource implications, and because G-O cancer has been the subject of NAEDI public awareness campaigns.

\section{Trial objectives}

The objectives of this trial are provided below:

1. To optimise an intervention based on the use of the G-O cancer eCDS tool, establish its acceptability, and collect relevant data to inform the design of a subsequent definitive phase III trial.

2. To obtain preliminary evidence on the effectiveness, implementation, and cost-effectiveness of the G-O cancer eCDS tool.

At the end of this phase II trial, we will have optimised the intervention and established its acceptability [25]. We will have also generated new knowledge on the process outcomes of the eCDS in primary care and its impact on resource utilisation and healthcare costs. In a subsequent phase III trial, we will examine the effect of the eCDS on G-O cancer stage at diagnosis, on surgical treatment, and on survival.

\section{Methods/design}

\section{Trial design}

This is a multi-site, phase II, cluster-randomised controlled trial (RCT), supported by the North Wales Organisation for Randomised Trials in Health (NWORTH Clinical Trials Unit (CTU)). Cluster randomisation is necessary because the intervention is implemented at the practice level, but some process measures and all outcomes relate to individual patients. Patients 55 years and older, presenting to their 
GP with symptoms associated with G-O cancer (NICE CG17, CG27, NG12), and capable of informed consent $[2,26,27]$ will be recruited from general practices, initially in the North East and North Cumbria and the Eastern Local Clinical Research Networks (LCRNs).

\section{Ethics}

Ethical approval for this study was granted on 7 November 2014 by the NRES Committee North East - Tyne \& Wear South (reference number 14/NE/1179). The study will fully comply with NHS Research Governance regulation. All necessary NHS and Durham University ethical approvals have been obtained. Informed consent will be obtained from all participants. Appropriate safety procedures will be followed by the researcher(s) when interviewing participants. Should any disclosures requiring action be made, the researchers will have access to the support of the project team.

\section{Study setting}

We will initially use two recruitment centres, in the North East and North Cumbria and in the Eastern LCRN areas to maximise population diversity with respect to socioeconomic status and to understand feasibility issues in diverse local health economies. Practices that have participated in the Macmillan Cancer Support eCDS initiative [20] and those with incompatible software will be excluded (i.e. practices without a SystmOne Clinical Computer System).

\section{Sample size}

This phase II trial is not powered to test specific hypotheses but to provide sufficient process data and enough participants with G-O cancer to provide estimates of effect to inform a phase III trial. We will recruit 60 practices with 1:1 randomisation, allocating 30 to each of the intervention and control arms. We anticipate that 64 patients (32 in each arm) who are subsequently diagnosed with G-O cancer will participate over the 16-month recruitment period, based on the following assumptions: (1) 17 out of every 1000 people more than 55 years of age undergo diagnostic gastroscopy for new upper gastrointestinal (GI) symptoms each year [1]; (2) $2.1 \%$ [7] to $5.5 \%$ (Trent Cancer Registry, personal communication) of these will have G-O cancer, depending on the route of referral (we have assumed $4 \%$ ); (3) the average practice size is 6500 , with $28 \%$ of the patients being older than 55 years of age [28], which implies that 1800 patients in each practice are age $55+$; (4) $80 \%$ of those with the index symptoms will be identifiable through READ codes (our own experience); and (5) the consent rate in the study population is $80 \%$. To illustrate the recruitment requirements, the number of patients with a new upper GI symptom annually ranges from 28-40/ 1000 [29]. Thus, from 30 control practices, we expect
1292-1843 participants, 780 diagnostic gastroscopies, and 32 patients with G-O cancer.

\section{Inclusion criteria}

To be included, patients must be 55 years of age or older and presenting to the GP with symptoms associated with G-O cancer $[10,27]$.

\section{Exclusion criteria}

Patients will be excluded according to the following criteria:

- If they are deemed unable to provide informed consent

- If the patient did not present to a GP with upper GI symptoms within the week before the search was run

- If a new prescription for a relevant medication has been made for reasons other than treatment of upper GI symptoms

- If the patient has had a diagnosis made or gastroscopy performed through a route that bypassed the GP.

\section{Practice recruitment}

GP practice recruitment will be supported via the Local Clinical Research Networks (LCRNs), who will approach practices in the designated geographical regions, favouring Research Site Initiative (RSI) practices. Recruitment will focus on RSI practices because these practices have an ongoing commitment to research and, generally, have allocated research nurse time. In practices that do not have funded research nurse time, a research nurse from the LCRNs, if possible, may be funded to support the study. Representatives from the LCRNs or study team will visit all practices interested in participating and deliver a short presentation covering the background, aims, and design of the trial. Patient recruitment will take place over 16 months or until the required number of participants has been obtained; whichever is shorter.

\section{Practice randomisation}

Practices that agree to participate will complete an initial assessment, sign a practice agreement form and will then be randomised into one of two conditions: usual diagnostic practice (control) and usual diagnostic practice plus access to the G-O Cancer electronic risk assessment tool (eRAT) (as the intervention).

Randomisation will be undertaken by the CTU via a secure, web-based, fully validated, customised system. The randomisation will be balanced using matched-pair methodology within region. Pairs of practices within a region will be presented for randomisation, which will be randomised on a 1:1 ratio. Practices are randomised in pairs to maintain allocation concealment. 


\section{Intervention}

Intervention practices will be provided with a modified version of the Macmillan eCDS tool on the BMJ Informatica platform, which will contain the G-O cancer eRAT (Fig. 1) embedded within the clinical system. This provides a drop-down box containing an interactive risk calculator, which can be opened at the GP's discretion during the consultation. It allows additional symptoms to be entered and generates a value for the risk of a currently undiagnosed G-O cancer. The GP then decides on further management, which may be clinical review in primary care, referral to a GI specialist, or direct referral for gastroscopy. The G-O cancer eCDS will also display an on-screen prompt at the start of a consultation if the relevant symptom(s) with a total risk $>2 \%$ has (have) been entered within the previous 12 months. Macmillan has undertaken extensive development and has addressed many of the key issues identified in the systematic reviews of eCDS, particularly the problem of prompt fatigue [23].

A clinician from the research team will also visit each intervention practice to deliver an implementation package developed from a review of relevant systematic reviews from the Cochrane Effective Practice and Organisation of Care Group, evidence on the barriers to implementation of decision-support tools in other disease areas, and normalisation process theory. This will include the same information as the presentation given to all practices by the LCRNs, along with specific details of how to use the eRAT. Within this, whilst emphasising the need to use clinical judgement in all cases, we will provide guidance on how to interpret the output from the eRAT based on the new NICE guidance for suspected cancer in primary care [27]. They may then wish to consider referral for patients who have a calculated risk of $3 \%$ or more, whilst those with a calculated risk of less than $3 \%$ may be better managed by review in primary care. This practice visit will then be supplemented by peerto-peer support as required.

\section{Control practices}

Patients presenting to the control practices with upper gastrointestinal (UGI) symptoms will experience the GP's usual diagnostic approach. Usual care is being used as a comparator for this study to help determine the actual benefits above normal practice. GPs in both arms of the trial will be offered free access to the Cancer Research UK-funded Royal College of General Practitioners (RCGP) online learning module on cancer diagnosis and to an end-of-study feedback event accredited for Continuing Professional Development (CPD) purposes. Control practices that have or acquire the Macmillan eCDS tool will be required to disable the G-O cancer functionalities for the duration of the trial.

\section{Process and outcome measures}

We will examine the following processes relating to the use of eCDS:

- Practitioner outcomes - the frequency and sustainability of the use of eRAT, the adherence to recommendations, and the attitudes to and role of eCDS

- Service outcomes - referral rates; use of diagnostic pathways (2WW and direct access gastroscopy, conversion (proportion of referrals with cancer diagnosis)) and detection rates (proportion of G-O cancers referred through these routes); time from the first consultation to diagnosis with cancer (diagnostic interval); and the stage of cancer at diagnosis using the TNM system

- Health economic outcomes based on estimates of resource use

- Patient outcomes - the acceptability of the use of eCDS.

We will perform the following tasks to inform the selection of outcome measures and power calculations for a subsequent Phase III trial:

1) Estimate the standard deviation for continuous outcomes

2) Estimate the proportions for binary outcomes (e.g. the proportion of patients referred in the control arm)

3) Estimate the recruitment and consent rates among those eligible for inclusion

4) Determine the feasibility of data extraction at follow-up

5) Examine how closely the stage at diagnosis is related to treatment with curative intent to inform choice of primary outcome measure.

\section{Data collection procedures Patient recruitment}

Practice administrative staff will use a pre-specified search string to search their electronic medical records weekly in order to identify patients who have presented to a GP within the previous week with qualifying symptoms. The records of patients thus identified will be reviewed by a designated individual (GP or practice research nurse) to determine eligibility to take part in the study. Administrative staff will post or use Docmail to send an information pack (consisting of invitation letter, participant information sheet, consent form and a reply paid envelope) to eligible patients. 


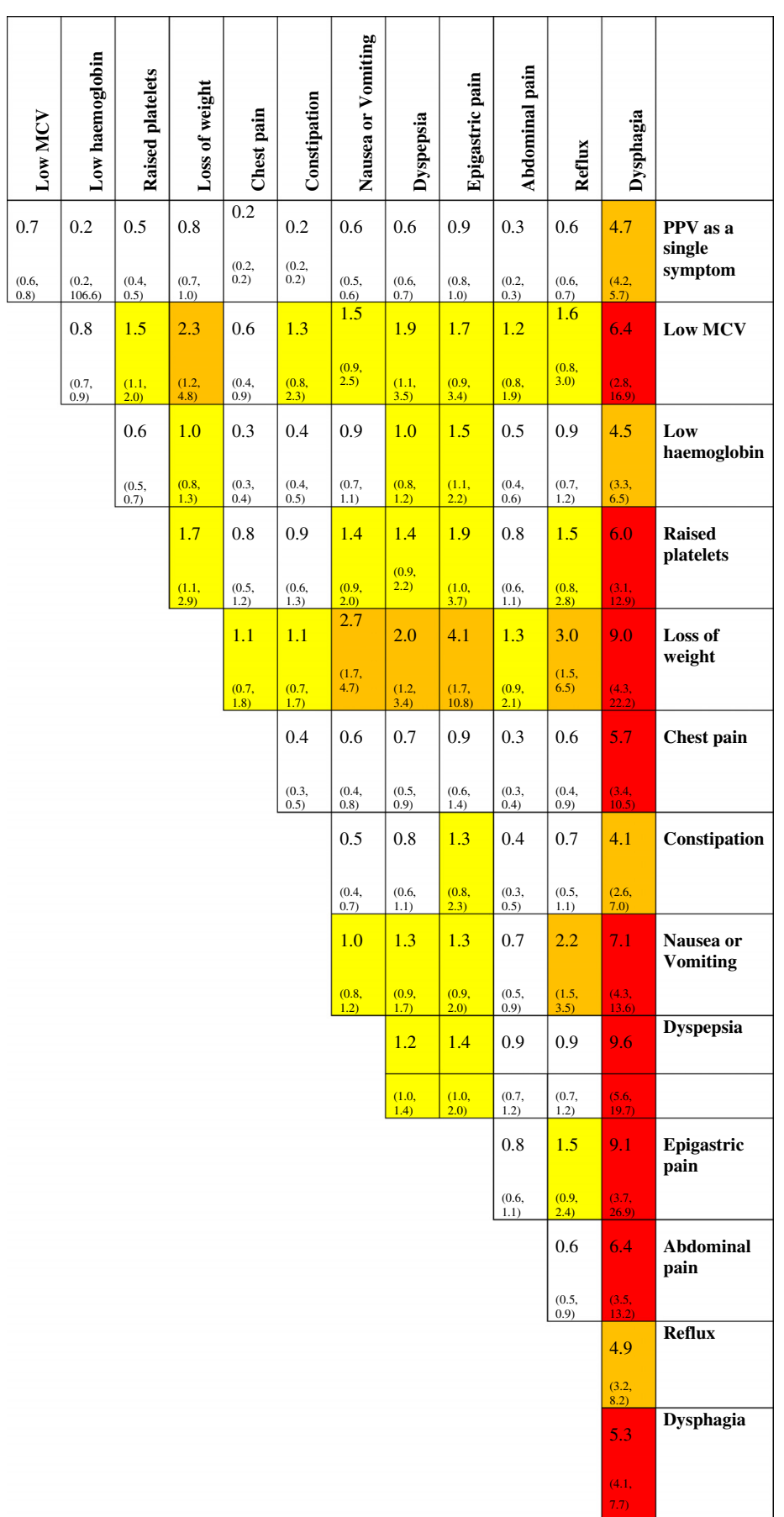

Fig. 1 Positive predictive values (95\% confidence intervals) for gastro-oesophageal cancer in men and women over 55 years of age for individual risk markers and for pairs of risk markers in combination. The top figure in each cell is the positive predictive value when both features are present. The two smaller figures represent the $95 \%$ Cls for the positive predictive value. These have not been calculated when any cell in the $2 \times 2$ table was below 5 (invariably this was because too few controls had both features). The yellow shading is for pairs of symptoms with a positive predictive value over $1 \%$, the amber shading is when the positive predictive value is above $2.0 \%$, and the red shading is for positive predictive values above $5.0 \%$. The cells along the diagonal relate to the positive predictive value when the same feature has been reported twice. Thus the back pain/back pain intersect is the positive predictive value for pancreatic cancer when a patient has attended at least twice with back pain

We will seek consent for access to primary and secondary care records for follow-up data and for qualitative interviews. Patients who wish to participate will return their completed consent forms to the research centre. The researcher will inform the practice if a consent form is received and whether consent was given for 
data to be used in the study. For those who consent to their data being used in the study, the practice will respond with the date of the index consultation and, in addition, for those in the intervention arm, will submit an age group (in 5-year increments of 55-59, 60-64, 65$69,70-74,75-79,80-84,85-89$, and $90+)$, the gender, and whether or not the eRAT was used.

A database of patients identified by the search string and those invited to participate will be kept by each practice; this database will be updated on a weekly basis. Practice administrative staff will identify any patients who have not responded at 2 weeks after being sent the invitation. These patients will be sent a reminder letter using either post or Docmail. Further information on the process of patient recruitment is provided in Table 1.

Patient consent is not being sought for the use of the eCDS during the consultation. This is because the eCDS tool is used at the discretion of the GP to support his/ her clinical decision making. This is consistent with previous randomised controlled trials of interventions in primary care [30, 31]. Use of the eCDS will not detrimentally affect patient care, as practitioners will still adhere to usual clinical guidelines.

\section{Data collection}

Academic research staff will visit GP practices every 6 months to extract data on participating patients to minimise any loss of data resulting from patient transfer or death. Staff will also visit gastroenterology units to extract data from secondary care patient records. All participants will be followed up for 6 months. Using data extraction templates, adapted from those developed by us for previous comparable studies, we will collect the information shown in Table 2.

Practice level data on direct-to-gastroscopy referral and conversion rates will also be collected. The East Midlands Knowledge and Intelligence Team will hold cancer waiting time data at the practice level on upper
Table 2 The type of data collection from the two care sites

Primary care data collection

Demographic data

Date of first consultation

Duration of index (first) consultation

Dates of subsequent consultations before referral

Referral in the episode of care $-\mathrm{Y} / \mathrm{N}$

Type of referral (2WW; open access gastroscopy; routine out-patient department; emergency, other)

Date of referral

Co-morbidities

RAT used - Y/N

Date used

Duration of consultation when RAT used

Final diagnosis

Secondary care data collection

Type of referral (2WW; OAG; routine OPD; emergency, other)

Diagnosis

Date of diagnosis

Stage

gastrointestinal (GI) 2WW referrals, conversion and detection rates, and emergency presentations.

\section{Nested qualitative study}

We will recruit GP/patient dyads at the intervention study sites to increase our understanding of the impact of eCDS use or non-use on the consultation. We will interview, within 6 weeks of consultation, up to $30 \mathrm{pa}-$ tients for whom the tool has been used for assessment of their symptoms, or where the tool was not used despite relevant symptoms, to gain a richer understanding of the impact of the use or non-use of the eCDS on the consultation. Consent will be sought from patients to be interviewed as part of the original participation consent

Table 1 Patient recruitment procedures

\begin{tabular}{lll}
\hline Step & Frequency & Person responsible \\
\hline Patient consults with relevant symptom & Weekly & Practice admin staff \\
Search of computer records to generate list of patients with qualifying symptoms & Weekly & Practice research nurse \\
List reviewed for eligibility and exclusions & Weekly & Practice admin staff \\
Invitations sent & & Researcher \\
Response from patient received by researcher & Weekly & Researcher \\
Consent information to practice & Weekly & Practice research nurse \\
Date of index consultation identified for each consenting patient sent to researcher & Weekly & Practice research nurse \\
Age group, gender, and use of eRAT $(\mathrm{Y} / \mathrm{N})$ identified for each consenting patient & & Practice admin staff \\
in the intervention practices sent to researcher & Weekly \\
Reminders sent & &
\end{tabular}


form. The research team will screen the patients who have consented to be interviewed to purposively sample for eRAT use and non-use. The researcher will also match consenting patients with their GP to identify GP/ patient dyads.

These GPs will have been provided with information about the nested qualitative study at the point of practice recruitment. GPs subsequently selected to take part in an interview will be contacted by telephone to confirm that they are willing to be interviewed and to arrange a suitable date and time for the interview to take place. GPs who are interested in participating will provide consent to participate. If these GPs are unable to find the time for a face-toface interview, they will be offered the option of a telephone interview. We will undertake semi-structured interviews with as many as possible of the GPs matched with their patients (up to 30).

We will also interview up to a further 10-15 GPs in order to identify and gain an understanding of the facilitators and constraints influencing the implementation and use of eCDS in clinical practice. GPs will be recruited purposively to sample as widely as possible (gender, age, trainer status, and frequency of eRAT use) and will include GPs in the intervention practices who did not use eCDS during the study. The interview schedule will be based on normalisation process theory.

At the end of their interview, all GPs will be asked if they are willing to take part in further interviews by telephone 3 months and 1 year later. If they agree to do so, they will be contacted after 2 months to confirm that they are still willing and to arrange a suitable date and time for the interview to take place.

Data provided by patients will remain confidential and will not be shared with their GP, and GP data will also not be shared with patients. Both patients and GPs will provide written consent prior to interviews commencing. GPs who are interviewed by telephone will be asked to provide verbal consent at the beginning of the interview and also complete a written consent form to be returned by post. Data will be audio-recorded and transcribed professionally.

\section{Data analysis \\ Statistical analysis}

Patient throughput will be summarised for the 16month recruitment period. For each trial arm, we will record the (1) number of eligible patients, (2) number of eligible patients approached to take part in the study, (3) number of eligible patients that consented/are recruited to the trial, and (4) number of recruited patients for whom outcome data are collected.

We will report the percentage of all eligible patients who are recruited (participation percentage) and the percentage of recruited patients for whom outcome data are collected with $95 \%$ confidence intervals. Separate reporting of the participation percentage by the trial arm will help identify any obvious recruitment bias resulting from randomising the practices before recruiting patients to the trial. However, we consider the risk of serious recruitment bias to be low because all eligible participants from intervention or control practices will be invited to participate, and this will be done by post after the index consultation, rather than during the consultation.

General practices in the intervention and usual-care arms will be described separately with respect to region (a factor used to balance the randomisation). Participating patients will be described separately within each trial arm with respect to relevant baseline demographic characteristics, using means and standard deviations (or medians and interquartile ranges) for continuous variables and percentages for categorical variables. No formal tests of significance will be used for these descriptive analyses.

It is not a primary objective of the study to obtain definitive estimates of the intervention effect on study outcomes. However, comparisons between the intervention and control arms using the intention-treat principle will be reported as ancillary analyses. A comparison of binary outcomes will be implemented using marginal logistic regression models using generalised estimating equations (GEEs) with information sandwich ('robust') estimates of standard error, specifying the correlation structure as exchangeable. In the case of rare binary events, the responses will be summarised using percentages only. Comparison of time-to-event outcomes will be carried out using marginal proportional hazards models with information sandwich ('robust') estimates of standard error.

Binary outcomes will be reported using a percentage for each trial arm, an odds ratio for comparing the trial arms, a $95 \%$ confidence interval, and $p$-value. Time-toevent outcomes will be presented as the hazard ratio, confidence interval, and $p$-value. Crude estimates of intervention effect and estimates adjusted for region and practice size will be presented. The following outcomes will be compared:

- For all patients

o Whether the patient is referred (binary)

- For all referred patients

o Whether the patient is referred via the 2 -week wait pathway (2WW) (binary)

o Whether patient is referred via the direct-access pathway (binary)

- Whether the referred patients were diagnosed with cancer (conversion - binary outcome)

- For all patients with cancer 
- Whether the diagnosed patients had been referred via the $2 \mathrm{WW} /$ direct-access pathway (binary outcome)

- For patients referred via the 2-week wait

- Cancer conversion rate (binary outcome)

- For patients referred via the direct-access pathway

- Cancer conversion rate - (binary outcome)

- For patients diagnosed with cancer

- Stage at diagnosis

- Treatment with curative intent (binary outcome),

- 1-year and 3-year survival

- Diagnostic interval (time from symptomatic presentation to cancer diagnosis)

\section{Health economic analyses}

First, we will map out the potential impacts of the eRAT tool from a health economic viewpoint by determining the places in the clinical pathway where the eRAT may impact the cost to the NHS and the benefit to patients (quality-adjusted life year (QALYs)). These will include the following:

- A change in the GP consultation length and use of eRATs that may impact GP costs

- A change in the stage at diagnosis and survival through earlier diagnosis that may impact QALYs and costs

- A change in the rates of diagnosis via primary care as opposed to emergency presentation that may impact diagnosis costs

- A change in the number of persons without cancer referred to secondary care that may impact diagnostic costs.

Second, we will estimated health economic outcomes. A simple health economic model will be developed using the eRAT impacts mapped out and populated by cost estimates obtained from published literature and other sources. Health economic outcomes generated will include the 2WW referral and gastroscopy costs associated with the eRAT; the cost per additional cancer case diagnosed; and the cost per emergency presentation avoided. We will also explore data requirements and possible modelling approaches for a health economic evaluation of a definitive trial.

\section{Qualitative analysis}

Our analyses will aim to provide a rich understanding of patient and practitioner views concerning the acceptability of eCDS use during the consultation and the potential effects on process, communication, and patient-centredness. We will use an iterative analytic process that will start near the beginning of data collection. Once the early findings have been reviewed, they will underpin the development of the thematic framework and inform potential adaptations to the interview schedule. The qualitative dataset will be fine-coded, and emergent themes will be identified using thematic analysis with a constant comparative approach [32] that will be aided by NVivo software. For the initial dyadic analysis, the experiences, views, and decisional processes of patients will be compared to those of their GP. The dyadic views will also be compared and contrasted across the dyadic dataset.

For the subsequent analyses of serial interviews with GPs over 12 months, the experiences, views, and decisional processes of each GP will be compared across time points, across GPs, and longitudinally to understand the facilitators and constraints influencing the implementation and use of eCDS in clinical practice.

\section{Data and documentation}

All data will be kept in accordance with the Data Protection Act 1998.

A database will be kept within each practice, which will contain information about patients invited to take part in this study; research teams will not have access to this information. The purpose of keeping this information is be able to identify non-responders and to exclude patients who re-consult about their G-O symptoms after already having been invited to take part in the study.

At Durham University and the University of Cambridge, all physical data will be stored in locked filing cabinets in a locked office in a password-protected corridor within the Wolfson Research Institute in Durham and the Department of Public Health and Primary Care respectively.

At both Durham and Cambridge Universities, all data stored electronically will be pseudo-anonymised and will be stored on password protected computers on secure servers.

The study team will have access to the final trial dataset.

\section{Data anonymisation}

All participants will be assigned an anonymous participant code, which will be used to pseudo-anonymise all data-collection forms. The key will be stored separately and will only be accessed by the local research team. Interviewees will be given a pseudonym, so they remain anonymous in the reporting of the qualitative data.

All personal data will be securely destroyed at the end of the study.

\section{Data monitoring}

Due to the non-medicinal and low-risk nature of the trial, a data monitoring committee will not be needed. The trial steering committee (which consists of an independent chair; the three lead applicants (GR, RDN, and 
FMW), the CTU lead (ZH), the trial statistician (OCU), a patient representative, an independent statistician, and an independent clinician) will meet every 6 months from the start of the study and will monitor study progress, approve a data analysis plan, and will ensure the study runs in accordance with the protocol and applicable standard operating procedures. Some members of the trial steering committee will take responsibility for the data monitoring and ethics.

The lead applicants will be responsible for communicating important protocol modifications to relevant parties. The trial is subject to the audit arrangements of the NIHR CRN. These are independent of the funder and the sponsor.

\section{Incidence of adverse events}

An adverse event will be defined as 'an event that arises directly from participation in the research', including complications that occur in the course of investigation. These will be reported using an adverse event reporting form.

\section{Dissemination policy}

For primary publications, the study team will form the basis of the writing committee and will also advise on any related publications. Primary publications will include members of the study team and other co-investigators as named authors or as part of a group authorship. In general, any related publications should include the principal investigators, lead researchers, and statistician as named authors; however, this is at the discretion of the writing committee.

\section{Discussion}

The objectives of this trial are to optimise the intervention and establish the acceptability of both the intervention and randomisation, confirm the suitability and selection of outcome measures, finalise the design for the phase III definitive trial, and obtain preliminary estimates of the intervention effect. These outputs need to be obtained before conducting a definitive trial, which would determine the effect on patient outcomes (the stage at diagnosis, treatment with curative intent, 1-year and 3-year survival, and diagnostic interval), resulting from the introduction of the eCDS into general practice for suspected G-O cancer.

We will use the Medical Research Council framework for the design and evaluation of complex interventions $[25,33,34]$. We are conducting a phase II, exploratory, cluster-randomised controlled trial to evaluate an eCDS tool for suspected G-O cancer (the e-RAT [3]).

The use of the eCDS in intervention practices may result in a change in practice activity levels for gastroscopy. The direction of effect is uncertain, since any increase in activity may be offset by fewer unnecessary referrals. An absolute $10 \%$ increase in gastroscopies in the intervention arm would constitute an additional 32 gastroscopies in each network area.

Public awareness campaigns for G-O cancer have been run as part of the Be Clear on Cancer campaign and resulted in an increase in GP consultations and referrals. If such a campaign runs in either or both of the two recruitment areas during the recruitment period, we will monitor and describe it.

New NICE guidance on the investigation of possible cancer was published in July 2015 after the study was designed and ethical approval was obtained but before recruitment had begun. The NICE guidance was based in part upon the research underpinning the eRAT, so control practices (if they fully adhere to the new guidance) may offer investigative practice closer in nature to the intervention practices than was expected at the time of the trial design. Adoption of the NICE guidelines (NG12) may also occur at different rates between the different clinical commissioning groups.

This is one of the first trials of eCDS for the early diagnosis of symptomatic cancer and will be completed in 2018.

\section{Trial status}

Site recruitment commenced in September 2015, and the trial is ongoing.

\section{Abbreviations \\ CPD: continuing professional development; CTU: clinical trials unit: eCDS: electronic clinical decision support; eRAT: electronic risk assessment tool; Gl: gastrointestinal; G-O: gastro-oesophageal; GP: general practitioner; LCRN: Local Clinical Research Network; NAEDI: National Awareness and Early Diagnosis Initiative; NICE: National Institute for Health and Care Excellence; NWORTH: North Wales Organisation for Randomised Trials in Health; QALY: quality-adjusted life year; RAT: risk assessment tool; RCGP: Royal College of General Practitioners; RCT: randomised controlled trial; RSI: Research Site Initiative; UGl: upper gastrointestinal; 2WW: 2-week wait.}

\section{Competing interests}

The authors declare that they have no competing interests.

\section{Authors' contributions}

GR, RDN, FMW, JE and WH conceived the research question and, with OCU and SW, designed the trial. OCU and ZH led the analytical design. GR, FMW, RDN, ZH, JUS, AK, and CN are responsible for management and delivery of the trial. JUS helped develop the implementation package and will lead the qualitative study on GP use and uptake of the tool. HJM and AT drafted the manuscript. All authors read and approved the final manuscript.

\section{Authors' information}

HJM: PhD, Post-Doctoral Research Associate

CN: BSc, MSc, PhD, MBPsS, Post-Doctoral Research Associate

AT: BSc, PhD, Post-Doctoral Research Assistant

JE: MA MBBCh FRACGP MRCGP DPhil. Herman Professor of Primary Care

Cancer Research and NHMRC Practitioner Fellow

WH: MD, FRCP, FRCGP, Professor of primary care diagnostics and GP. Was

clinical lead in the production of the NICE 2015 guidelines, and was a

co-author in the study that produced the eRAT.

ZH: PhD, CStat, CSci. Principal Statistician at NWORTH (North Wales

Organisation for Randomised Trials in Health (and Social Care))

AK: MB BChir MPH, Research Associate

RDN: MB ChB PhD FRCGP, Professor of Primary Care Medicine. Chair of the NCRI Primary Care Clinical Studies Group.

OCU: PhD, Associate Professor in Medical Statistics (trial statistician) 
JUS: MB BChir PhD MRCGP, Clinical Lecturer in General Practice FMW: MA, MD, FRCGP, Principal Researcher in Primary Care Cancer Research, NIHR Clinician Scientist, and Chair of the NCRI Primary Care Clinical Studies Group's Early Diagnosis sub-group.

SW: MMath, PhD (Pure Mathematics), research fellow in cost-effectiveness modelling, Health Economics and Decision Science Section

GR: FRCGP, FRCP, Professor of General Practice and Primary Care

\section{Acknowledgements}

OCU is supported by the National Institute for Health Research (NIHR) Collaboration for Leadership in Applied Health Research and Care South West Peninsula. The views expressed are those of the author(s) and not necessarily those of the NHS, the NIHR, or the Department of Health. Dr. Nicola Hall contributed to the initial set-up phase of the trial.

\section{Sources of funding}

HJM is an employee of Durham University.

$\mathrm{CN}$ is an employee of Durham University and is funded by the ECASS trial. AT is an employee of Durham University.

JE is an employee of the University of Melbourne and is supported through an NHMRC Practitioner Fellowship.

$\mathrm{WH}$ is an employee of the University of Exeter. He is part-funded by the National Institute for Health Research (NIHR) Collaboration for Leadership in Applied Health Research and Care South West Peninsula at the Royal Devon and Exeter NHS Foundation Trust. The views expressed are those of the author(s) and not necessarily those of the NHS, the NHHR or the Department of Health.

$\mathrm{ZH}$ is an employee of Bangor University and is part-funded by Health and Care Research Wales.

AK is an employee of Cambridge University.

RDN is an employee of Bangor University. He is part funded by the Betsi Cadwaladr University Health Board and Public Health Wales.

OCU is an employee of the University of Exeter and is supported by the National Institute for Health Research (NIHR) Collaboration for Leadership in Applied Health Research and Care South West Peninsula.

JUS is funded by a National Institute for Health Research (NIHR) Clinical Lectureship.

FMW is funded by a National Institute for Health Research (NIHR) Clinician Scientist award. The views expressed are those of the author(s) and not necessarily those of the NHS, the NIHR, or the Department of Health. SW is an employee of the School of Health and Related Research, University of Sheffield.

GR is an employee of Durham University.

\section{Funding source}

Cancer Research UK, Reference number: C6971/A17940. Cancer Research UK is not involved in the research other than to provide funding.

The trial sponsor is Durham University (protocol version 4.0, dated 13 July 2015).

\section{Author details}

'School of Medicine, Pharmacy \& Health, Durham University, Wolfson Research Institute, Queens Campus, Thornaby TS17 6BH, UK. ${ }^{2}$ General Practice and Primary Health Care Academic Centre, The University of Melbourne, 200 Berkeley St, Carlton, VIC 3053, Australia. ${ }^{3}$ The Veysey Building, University of Exeter, College House, Exeter EX1 2LU, UK. ${ }^{4}$ NWORTH Clinical Trials Unit, Bangor University, Y Wern, Normal Site, Holyhead Road, Bangor LL57 2PZ, UK. ${ }^{5}$ Primary Care Unit, Department of Public Health and Primary Care, University of Cambridge, 2 Worts Causeway, Cambridge CB1 8RN, UK. ${ }^{6}$ North Wales Centre for Primary Care Research, Bangor University, Gwenfro Units 4-8, Wrexham Technology Park, Wrexham LL13 7YP, UK. ${ }^{7}$ NIHR CLAHRC South West Peninsula (PenCLAHRC), University of Exeter, South Cloisters Building, St Luke's Campus, Heavitree Road, Exeter EX1 2LU, UK. ${ }^{8}$ School of Health and Related Research, University of Sheffield, Regent Court, 30 Regent Street, Sheffield S1 4DA, UK.

\section{Received: 6 January 2016 Accepted: 19 March 2016}

\section{Published online: 04 April 2016}

\section{References}

1. Hamilton W. Cancer diagnosis in primary care. Br J Gen Pract. 2010;60(571): 121-8. doi:10.3399/bjgp10X483175.
2. National Institute for Health and Care Excellence. Referral guidelines for suspected cancer. [CG27]. 2005.

3. Stapley S, Peters TJ, Neal RD, Rose PW, Walter FM, Hamilton W. The risk of oesophago-gastric cancer in symptomatic patients in primary care: a large case-control study using electronic records. Br J Cancer. 2013;108(1):25-31. doi:10.1038/bjc.2012.551.

4. Bowrey DJ, Griffin SM, Wayman J, Karat D, Hayes N, Raimes SA. Use of alarm symptoms to select dyspeptics for endoscopy causes patients with curable esophagogastric cancer to be overlooked. Surg Endosc. 2006;20(11):1725-8. doi:10.1007/s00464-005-0679-3.

5. Lyratzopoulos G, Neal RD, Barbiere JM, Rubin GP, Abel GA. Variation in number of general practitioner consultations before hospital referral for cancer: findings from the 2010 National Cancer Patient Experience Survey in England. Lancet Oncol. 2012;13(4):353-65. doi:10.1016/s14702045(12)70041-4.

6. Elliss-Brookes L, McPhail S, Ives A, Greenslade M, Shelton J, Hiom S, et al. Routes to diagnosis for cancer-determining the patient journey using multiple routine data sets. Br J Cancer. 2012;107(8):1220-6. doi:10.1038/bjc.2012.408.

7. Shawihdi M, Thompson E, Kapoor N, Powell G, Sturgess RP, Stern N, et al. Variation in gastroscopy rate in English general practice and outcome for oesophagogastric cancer: retrospective analysis of hospital episode statistics. Gut. 2014;63(2):250-61. doi:10.1136/gutjnl-2012-304202.

8. Muir Gray, Erica Ison (Eds). NHS atlas of variation 2011 Map 42: rate of activity for gastroscopy (upper Gl endoscopy) per population by PCT. Right Care. 2012

9. Bisschops R, Wilmer A, Tack J. A survey on gastroenterology training in Europe. Gut. 2002;50(5):724-9.

10. Hamilton W, Peters T, Round A, Sharp D. What are the clinical features of lung cancer before the diagnosis is made? A population based case-control study. Thorax. 2005;60(12):1059-65. doi:10.1136/thx.2005.045880.

11. Hamilton W, Round A, Sharp D, Peters TJ. Clinical features of colorectal cancer before diagnosis: a population-based case-control study. $\mathrm{Br} J$ Cancer. 2005;93(4):399-405.

12. Hippisley-Cox J, Coupland C. Identifying patients with suspected gastrooesophageal cancer in primary care: derivation and validation of an algorithm. Br J Gen Pract. 2011;61(592):e707-14. doi:10.3399/bjgp11X606609.

13. Hippisley-Cox J, Coupland C. Identifying patients with suspected colorectal cancer in primary care: derivation and validation of an algorithm. $\mathrm{Br} J \mathrm{Gen}$ Pract. 2012;62(594):e29-37. doi:10.3399/bjgp12X616346.

14. Hippisley-Cox J, Coupland C. Identifying patients with suspected pancreatic cancer in primary care: derivation and validation of an algorithm. $\mathrm{Br} J$ Gen Pract. 2012;62(594):e38-45. doi:10.3399/bjgp12X616355.

15. Stapley S, Peters TJ, Neal RD, Rose PW, Walter FM, Hamilton W. The risk of pancreatic cancer in symptomatic patients in primary care: a large casecontrol study using electronic records. Br J Cancer. 2012;106(12):1940-4. doi:10.1038/bjc.2012.190.

16. Hamilton W. The CAPER studies: five case-control studies aimed at identifying and quantifying the risk of cancer in symptomatic primary care patients. Br J Cancer. 2009;101 Suppl 2:S80-6. doi:10.1038/sj.bjc.6605396.

17. Hippisley-Cox J, Coupland C. Symptoms and risk factors to identify men with suspected cancer in primary care: derivation and validation of an algorithm. Br J Gen Pract. 2013;63(606):e1-10. doi:10.3399/bjgp13X660724.

18. Hippisley-Cox J, Coupland C. Symptoms and risk factors to identify women with suspected cancer in primary care: derivation and validation of an algorithm. Br J Gen Pract. 2013;63(606):e11-21. doi:10.3399/bjgp13X660733.

19. Hamilton W, Green T, Martins T, Elliott K, Rubin G, Macleod U. Evaluation of risk assessment tools for suspected cancer in general practice: a cohort study. $\mathrm{Br} J$ Gen Pract. 2013;63(606):e30-6. doi:10.3399/bjgp13X660751.

20. Dikomitis L, Green T, Macleod U. Macmillan Electronic Risk Assessment Tool Summary. Dealing with uncertainty: a qualitative evaluation of the usability and acceptability of an electronic risk assessment tool to aid cancer diagnosis in general practice. 2012. p. 1-19.

21. Kawamoto K, Houlihan CA, Balas EA, Lobach DF. Improving clinical practice using clinical decision support systems: a systematic review of trials to identify features critical to success. BMJ. 2005;330(7494):765. doi:10.1136/ bmj.38398.500764.8F

22. Garg AX, Adhikari NJ, McDonald H, et al. Effects of computerized clinical decision support systems on practitioner performance and patient outcomes: a systematic review. JAMA. 2005:293(10):1223-38. doi:10.1001/jama.293.10.1223.

23. Roshanov PS, Fernandes N, Wilczynski JM, Hemens BJ, You JJ, Handler SM et al. Features of effective computerised clinical decision support systems: metaregression of 162 randomised trials. BMJ. 2013;346. doi:10.1136/bmj.f657 
24. Bryan C, Boren SA. The use and effectiveness of electronic clinical decision support tools in the ambulatory/primary care setting: a systematic review of the literature. Inform Prim Care. 2008;16(2):79-91.

25. Campbell NC, Murray E, Darbyshire J, Emery J, Farmer A, Griffiths F, et al. Designing and evaluating complex interventions to improve health care. $\mathrm{Br}$ Med J. 2007;334(7591):455-9. doi:10.1136/bmj.39108.379965.BE.

26. National Institute for Health and Care Excellence. Dyspepsia: management of dyspepsia in adults in primary care. [CG17]. 2004.

27. National Institute for Health and Care Excellence. Suspected cancer: recognition and referral. [NG12]. 2015.

28. Office for National Statistics. 2011 Census, population and household estimates for England and Wales. 2013.

29. Thompson WG, Heaton KW, Smyth GT, Smyth C. Irritable bowel syndrome in general practice: prevalence, characteristics, and referral. Gut. 2000;46(1):78-82.

30. Downs M, Turner S, Bryans M, Wilcock J, Keady J, Levin E, et al. Effectiveness of educational interventions in improving detection and management of dementia in primary care: cluster randomised controlled study. Br Med J. 2006;332(7543):692-6.

31. Flottorp S, Oxman AD, Havelsrud K, Treweek S, Herrin J. Cluster randomised controlled trial of tailored interventions to improve the management of urinary tract infections in women and sore throat. BMJ. 2002;325(7360):367.

32. Braun V, Clarke V. Using thematic analysis in psychology. Qual Res Psychol. 2006;3(2):77-101.

33. Craig P, Dieppe P, Macintyre S, Michie S, Nazareth I, Petticrew M. Developing and evaluating complex interventions: the new Medical Research Council guidance. BMJ. 2008;337. doi:10.1136/bmj.a1655

34. Foster $\mathrm{N}$, Little $\mathrm{P}$. Methodological issues in pragmatic trials of complex interventions in primary care. Br J Gen Pract. 2012;62(594):10-1. doi:10.3399/ bjgp12X616238.

\section{Submit your next manuscript to BioMed Central and we will help you at every step:}

- We accept pre-submission inquiries

- Our selector tool helps you to find the most relevant journal

- We provide round the clock customer support

- Convenient online submission

- Thorough peer review

- Inclusion in PubMed and all major indexing services

- Maximum visibility for your research

Submit your manuscript at www.biomedcentral.com/submit 